\title{
Damage detection based on damping analysis of ambient vibration data
}

\author{
Michele Frizzarin ${ }^{1, *, \dagger}$, Maria Q. Feng ${ }^{2}$, Paolo Franchetti ${ }^{1}$, Serdar Soyoz ${ }^{2}$ \\ and Claudio Modena ${ }^{1}$ \\ ${ }^{1}$ Department of Structural and Transportation Engineering, University of Padova, Italy \\ ${ }^{2}$ Department of Civil and Environmental Engineering, University of California, Irvine, U.S.A.
}

\begin{abstract}
SUMMARY
Enabling an automated, remote and rapid detection of structural damage, sensor-based structural health monitoring is becoming a powerful tool for maintenance of civil engineering structures. In this study, a baseline-free, time-domain damage detection method was developed for concrete structures, which is based on analysis of nonlinear damping from measured structural vibration responses. The efficacy of the proposed method was demonstrated through a large-scale concrete bridge model subjected to different levels of seismic damage caused by shaking table tests. By applying the random decrement signature technique, the proposed method successfully identified, from its ambient vibration responses, nonlinear damping of the bridge associated with the seismic damage. The amount of the nonlinear damping increases as the seismic damage becomes more severe. This paper also compares the damage detection results with those obtained by stiffness-based methods, demonstrating a strong correlation between the increase in nonlinear damping and the decrease in structural stiffness associated with the increase in damage severity. Copyright (C) 2008 John Wiley \& Sons, Ltd.
\end{abstract}

KEY WORDS: vibration measurement; seismic damage detection; nonlinear damping; baseline-free; ambient vibration; random decrement; shaking table test; reinforced concrete structure; bridge

\section{INTRODUCTION}

The work presented in this paper deals with structural dynamics and develops theoreticalexperimental identification techniques for structural damage detection. The ultimate goal is to detect, locate, and assess, in real-time and at a remote location, bridge structural damage, by processing vibration responses of a bridge measured by on-bridge sensors. This is particularly

\footnotetext{
*Correspondence to: Michele Frizzarin, Department of Structural and Transportation Engineering, University of Padova, Italy.

†E-mail: michele.frizzarin@gmail.com
}

Contract/grant sponsor: California Department of Transportation (Caltrans); contract/grant number: 59A0311

Contract/grant sponsor: Federal Highway Administration; contract/grant number: DTFH61-07-C- 00031

Copyright (C) 2008 John Wiley \& Sons, Ltd.

Received 9 February 2008

Revised 20 May 2008

Accepted 5 October 2008 
valuable for post-seismic damage detection by improving the current visual inspection, which is time consuming, subjective, and potentially dangerous for inspectors.

Research on vibration-based damage detection began in late 1970s in aerospace structures [1-3]. Then in the early 1980s, the research emerged to the civil engineering structures, especially for bridges and monumental structures. Many system identification techniques and damage detection methods have been developed over the past 30 years. Some full-scale tests were conducted, but researchers had difficulties to completely validate the efficacy of the proposed methods, mainly due to the unavailability of undamaged structures as a baseline. Of considerable interest was the research on large civil engineering structures developed since 1995 at the Los Alamos National Laboratory, as it allowed researchers to compare the dynamic response of a structure before and after the introduction of different levels of damage [4,5]. Most of these studies were based on changes in the dynamic characteristics of structures, since changes in physical properties cause detectable changes in modal parameters [6,7]. Usually these techniques are based on the measurement of changes in dynamic parameters including natural frequencies, mode shapes, and damping ratios. They are based on linear analysis, and their reliability and application range are widely known [8].

The main issue that must be addressed, when the frequency-domain modal identification techniques are applied for seismic damage detection, is the presence of nonlinearity in the structural response. Most of the methods developed so far are based on frequency-domain analysis, which assumes linear structural responses. In case of civil engineering structures, this assumption is difficult to accept. As suggested by some studies [9,10], a nonlinear analysis is necessary during extreme events, because most civil engineering structures exhibit nonlinear response to strong excitations such as destructive earthquakes.

A major advantage of time-domain-based methods over the frequency domain ones is its ability to handle nonlinear structural responses. However, most of time-domain methods are based on the comparison between damaged and undamaged states [11]. In other words, an undamaged baseline is required. Often, the lack of the baseline can make the methods impractical for applications. At best, some of these methods might offer detection of damage/ deterioration between the current condition and a future condition. However, this would require testing the structure to form a set of baseline data and having a good knowledge of the current defects of the structure. Very few studies have been performed on damage assessment without explicit reference to the undamaged baseline [12].

In this paper, the authors develop a damage detection method originally proposed by Modena et al. based on time history analysis [13-16]. It uses nonlinear damping as a damage index for reinforced concrete structure elements. In particular, the proposed technique can predict the presence of damage in a structural element without any reference to its undamaged baseline, using its free vibration (or impulse response). In this study, the method is developed, for the first time, for applications in seismic damage detection of a concrete bridge structure using its ambient vibration response. The large-scale three-bent bridge has been previously studied at the University of California, Irvine, through seismic shaking table tests for seismic damage detection using structural stiffness as a damage index $[17,18]$. The realistic bridge model and its realistic seismic damage at different levels caused by progressive seismic shaking enables a deep and complete test of the efficacy of the proposed damage detection method.

In particular, this study proposes the use of the random decrement (RD) signature technique that enables the application of the proposed damage detection method from the decayed free vibration signals to random responses. Ambient vibrations are the most accessible data that can 
be acquired from a real structure, since the measurement requires neither the structure being taken out of service, nor expensive exogenous excitations.

\section{NONLINEAR DAMPING ON REINFORCED CONCRETE STRUCTURES}

Experiments carried out in the late 1990s on pre-cast reinforced concrete panels [14] showed that the presence of a small, visually undetectable crack caused negligible changes in natural frequencies, but a considerable increase in damping. The depth of the studies led to the conclusion that a clear relationship exists between the presence and the entity of damage in the structures and the appearance of a nonlinear dissipative mechanism, which can be expressed by means of a nonlinear damping. As many studies outlined [19], nonlinear damping in reinforced concrete can be directly correlated to the presence of cracks.

There is a fundamental difference between damage detection methods based on modal parameters (frequencies, mode shapes, damping) and the ones based on identification of anomalies, e.g. nonlinearity. In the methods based on classical modal parameters, damage is revealed though a difference in behavior between undamaged and damaged conditions. Therefore, these methods have difficulties to detect damage if the characteristics of the structure in the undamaged state are not available. On the contrary, in case of the methods based on nonlinearity, the anomaly itself reveals the damage, without any reference to the undamaged state [14]. This latter approach has not been extensively studied in literature for damage detection purposes, although many researchers have studied the nonlinear damping phenomenon [19-22].

\subsection{Theoretical background}

Damping in a vibrating structure is associated with a dissipation of mechanical energy [19]. The energy dissipation equals the work done by the damping force. In case of a free vibration the presence of damping results in a continuous decay of the amplitude. If the motion is an oscillation, for every cycle the quantity of energy loss $\Delta E$ can be expressed as

$$
\Delta E=\oint \sigma \mathrm{d} \varepsilon
$$

where $\sigma$ is the stress (or internal force) and $\varepsilon$ is the strain (or deformation). This quantity can be represented as the area inside the hysteretic loop formed for each cycle.

If the system is modeled as a simple linear oscillator, the differential equation of motion is expressed as

$$
m \ddot{x}+c \dot{x}+k x=F(t)
$$

where $x$ is the displacement, $m$ is the mass of the system, $k$ is the stiffness, and $c$ is the damping coefficient, while $F(t)$ is the external excitation. Many researches proved how, in the undamaged condition, the dissipation of energy is due mostly to material damping, which appears macroscopically viscous, i.e. proportional to the velocity of motion [20]. The energy dissipated per cycle becomes

$$
\Delta E_{\mathrm{visc}}=\int_{0}^{T}(c \dot{x}) \dot{x} \mathrm{~d} t=\pi c \omega x_{0}^{2}
$$


where $\omega$ is the natural frequency of the system, $x_{0}$ is the initial amplitude of oscillation, and the integral is taken over a period $T$.

A viscous damping ratio can be defined as

$$
\xi=\frac{1}{4 \pi} \frac{\Delta E_{\mathrm{visc}}}{E_{\mathrm{pot}}}
$$

where $E_{\text {pot }}$ is the maximum strain potential energy of the structure

$$
E_{\mathrm{pot}}=\frac{1}{2} k x_{0}^{2}
$$

In case of free vibration, from Equations (3)-(5) the viscous damping ratio becomes

$$
\xi=\frac{c}{2 m \omega}
$$

This quantity is independent from the amplitude of oscillation $x_{0}$.

In reality, civil engineering structures often do not show purely viscous damping. In particular, the behavior of reinforced concrete elements is strongly influenced by cracking. If the element is damaged, within the cracks the most significant dissipation mechanism can be represented, with the best agreement to the real behavior, with Coulomb friction. This is due to the phenomenon of slip between steel and concrete.

The differential equation that describes a purely friction-damped system is

$$
m \ddot{x}+F_{\mathrm{C}} \frac{\dot{x}}{|\dot{x}|}+k x=F(t)
$$

where $F_{\mathrm{C}}$ is the friction force. Equation (7) is nonlinear, and its solution needs to be calculated through numerical integration. Usually $F_{C}$ is expressed with reference to the compression force $N$ acting between the surfaces, such as

$$
F_{\mathrm{C}}=\mu N
$$

where $\mu$ is the friction coefficient, which depends only on the contact materials.

The energy loss per cycle for this model, with reference to Equation (1), becomes

$$
\Delta E_{\text {frict }}=\int_{0}^{T} F_{\mathrm{C}} \frac{\dot{x}}{|\dot{x}|} \dot{x} \mathrm{~d} t=4 F_{\mathrm{C}} x_{0}
$$

An equivalent frictional damping ratio can be therefore defined as

$$
\gamma=\frac{1}{4 \pi} \frac{\Delta E_{\text {frict }}}{E_{\text {pot }}}=\frac{2 F_{\mathrm{C}}}{\pi k x_{0}}
$$

Unlike the viscous damping ratio, this parameter is dependent on the initial amplitude $x_{0}$. Figure 1 shows a comparison between the hysteretic loops obtained from the two models of viscous and friction damping, respectively. Unlike the viscous damping, the shape of the hysteretic loop of the friction system is due to the fact that the damping force has a constant intensity, always opposite to the direction of motion, as seen in Equation (7).

The solutions of Equations (2) and (7), in case of free vibration, are represented in Figure 2.

The envelopes of these decays $a(t)$ represented in Figure 2 can be expressed, respectively as

$$
\begin{gathered}
a(t)=x_{0} \mathrm{e}^{-\xi \cdot \omega \cdot t} \text { for viscous damping } \\
a(t)=x_{0}\left(1-\frac{2}{\pi} \frac{x_{\lim }}{x_{0}} \omega t\right) \text { for friction damping }
\end{gathered}
$$




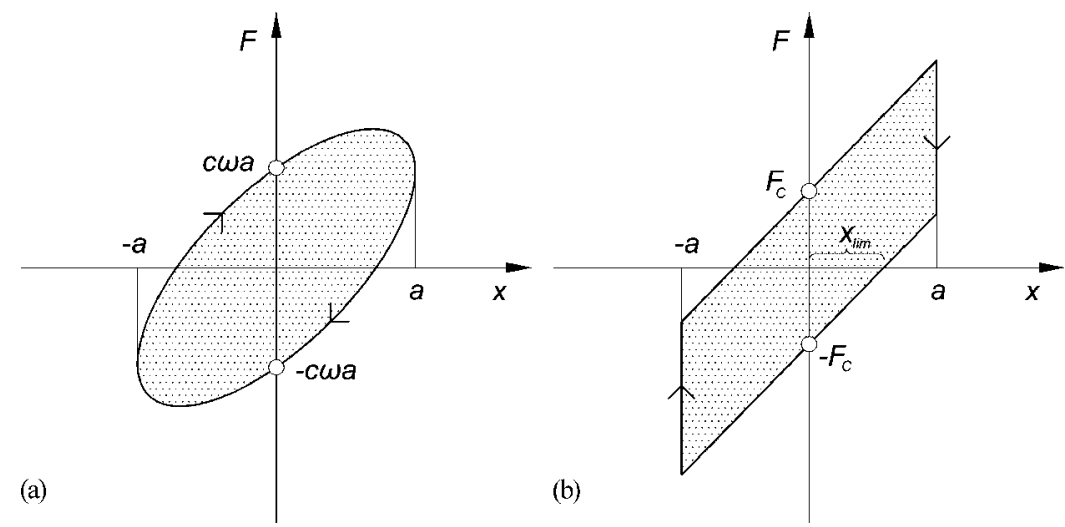

Figure 1. Hysteretic loops for (a) purely viscous damper and (b) purely friction damper.

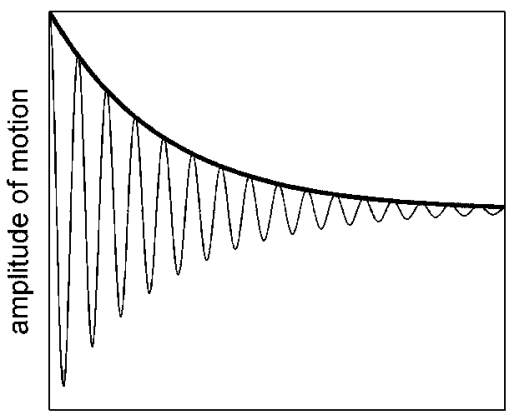

time

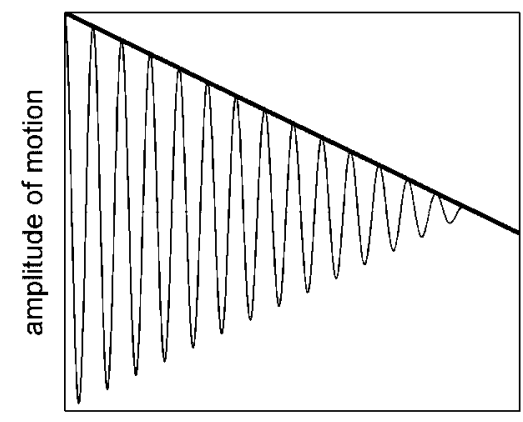

time

Figure 2. Free decay for (a) purely viscous damper and (b) purely friction damper.

where $x_{\lim }$ is the limit displacement in static equilibrium, defined as

$$
x_{\lim }=\frac{F_{\mathrm{C}}}{k}
$$

From Equations (10) and (13) the frictional damping ratio $\gamma$ can be expressed as

$$
\gamma=\frac{2 x_{\lim }}{\pi x_{0}}
$$

Therefore, Equation (12) becomes

$$
a(t)=x_{0}(1-\gamma \omega t)
$$

Considering its complexity, a cracked bending element, where both the viscous and friction damping phenomena co-exist at the same time, can be modeled as a combined system. In particular, Figure 3 shows that in the cracked zone where there is friction in the reinforcement surface, the most significant dissipation mechanism is the friction damping. On the contrary, in the compression zone, it can be assumed that only material (viscous) damping is present. Therefore, the bending element can be modeled as shown in Figure 3, where $k$ represents the bending stiffness of the element, while $m$ the relevant mass. 

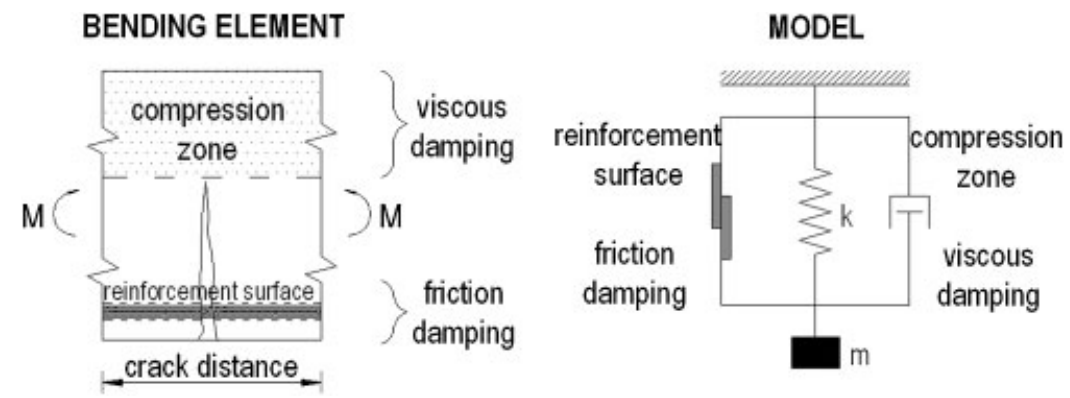

Figure 3. Cracked bending element and corresponding model.

The mass-normalized equation of motion of the combined model becomes

$$
\ddot{x}+2 \omega \xi \dot{x}+\omega^{2} x_{\lim } \frac{\dot{x}}{|\dot{x}|}+\omega^{2} x=0
$$

Equation (16) is nonlinear and its exact solution can be found through a numerical integration [22]. A sufficiently accurate approximation for practical purposes was obtained, assuming that the total loss of energy $\Delta E_{\text {tot }}$ can be interpreted as a simple sum of the viscous and the friction dissipations [16]. If no external forces act on the system (i.e. free vibration), $\Delta E_{\text {tot }}$ is equal to the variation of potential energy of the system, i.e.

$$
\Delta E_{\mathrm{pot}}=\Delta E_{\mathrm{visc}}+\Delta E_{\text {frict }}
$$

In presence of a Coulomb friction mechanism, free motion is generally non-sinusoidal. However, a sinusoidal solution of Equation (16) is acceptable when elastic forces are greater than friction forces. In this case, the three terms in Equation (17) can be replaced with Equations (3), (5), and (9), this time considering $x$ instead of $x_{0}$, since the balance is not yet integrated in time

$$
\Delta\left(\frac{1}{2} \omega^{2} x^{2}\right)=2 \pi \xi \omega^{2} x^{2}+4 \omega^{2} x_{\lim } x
$$

The same balance can be expressed in terms of power, dividing each member by $\Delta t=T=2 \pi / \omega$, and obtaining

$$
\frac{\Delta x}{\Delta t}=\xi \omega x+\frac{2}{\pi} \omega x_{\lim }
$$

By integrating Equation (19) over a period the solution of Equation (16) can be calculated. Its envelope can be expressed, considering Equations (6) and (14), as a function of the initial amplitude $x_{0}$, the natural frequency of the system $\omega$ and the two damping ratios $\xi$ for viscous damping and $\gamma$ for friction damping:

$$
a(t)=x_{0}\left[\left(1+\frac{\gamma}{\xi}\right) \mathrm{e}^{-\xi \cdot \omega \cdot t}-\frac{\gamma}{\xi}\right]
$$

Figure 4 shows the free decay of the described combined model, as defined in Equation (20).

The values of $\gamma$ and $\xi$ will give information about the percentage of the total energy dissipated by each damping phenomenon. $\gamma=0$ means that no friction damping acts on the system: therefore no cracks are present. On the contrary, a positive value of $\gamma$ means that a frictional dissipation acts on the element. Therefore, the friction damping ratio $\gamma$ can be directly correlated to the presence of damage in the considered concrete element. 


\subsection{Damage detection procedure}

The proposed procedure for damage detection starts from the analysis of a free vibration of the considered structure, given as a response to an impulse excitation. A free mono-frequency signal needs to be extracted from the response of the structure.

After obtaining the single degree of freedom (SDOF) free response, the envelope of the decay needs to be derived, by picking the oscillation peaks. For every period of oscillation two values - positive and negative peak - are extracted, by simply considering the opposite value of the negative peaks.

The damage detection method is based on the fact that the envelope of a free decay gives information about the dissipation forces acting on the system. Depending on the shape of the envelope, it can be concluded that either only viscous dissipation acts on the system-pure exponential decay, as shown in Figure 2(a), or that both viscous and friction forces are present-combined decay, as in Figure 4. In order to assess which of the described models is more appropriate to represent the recorded signal, the envelope of the oscillation must be fitted with both Equations (11) and (20). The values of $\xi$ and $\gamma$ can thus reveal information about the damping mechanisms.

Figures 5 and 6 show the fitting procedure applied in a previous study on pre-cast reinforced concrete panels [16]. In particular, Figure 6 shows the different shapes of the oscillation in case of undamaged and damaged elements. The damping characteristics can be visually described from a semi-logarithmic plot of the decay: a straight line can be directly correlated to a viscous

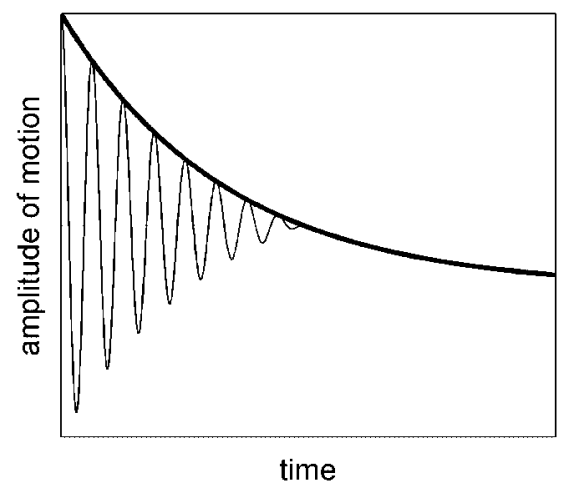

Figure 4. Free decay for the viscous and friction combined model.
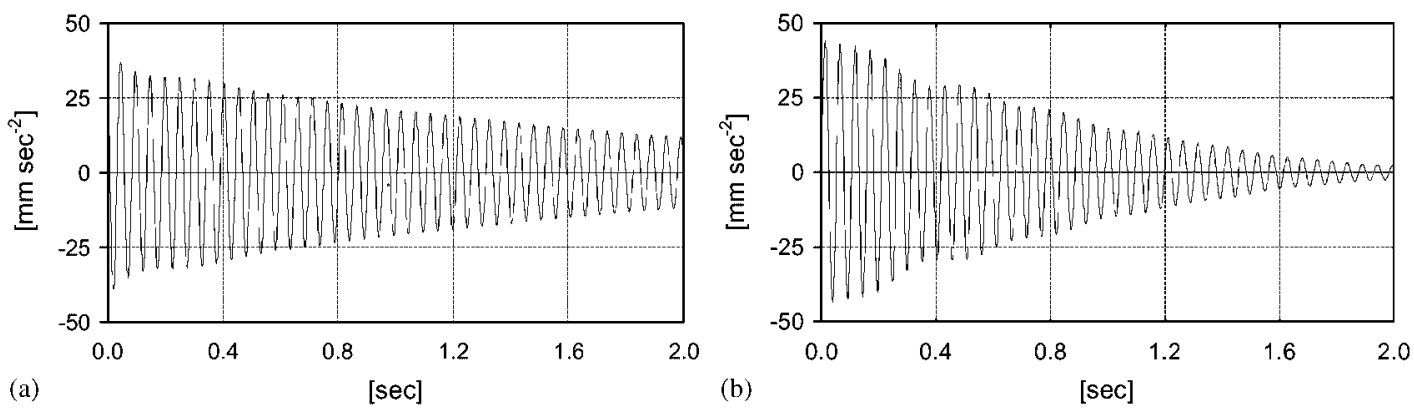

Figure 5. Time history free response of an undamaged (a) and cracked (b) panel: filtered signal. 

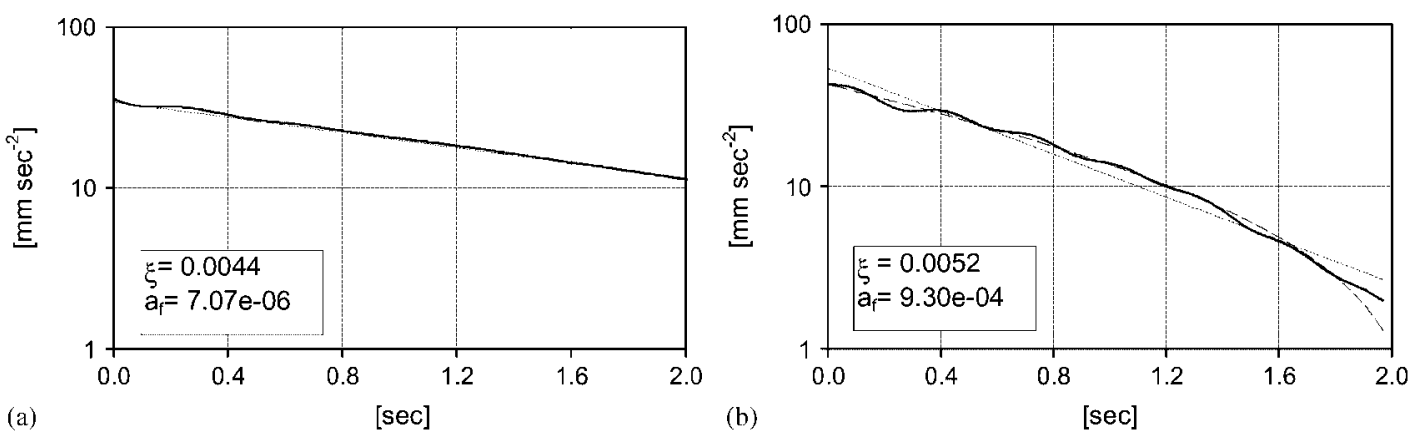

Figure 6. Undamaged (a) and cracked (b) panels: envelopes of the oscillations (solid line), exponential fit (dotted line) and combined fit (dashed line).

decay (Figure 6(a)), while a negative curvature is proof of the presence of a combined mechanism (Figure 6(b)).

\section{AMBIENT VIBRATION RESPONSES: USE OF THE RD SIGNATURE}

As presented in Section 2, the nonlinear damping-based damage detection method was originally developed to use free vibration or impulse response and applied to small concrete specimens tested in laboratory conditions. For large-scale concrete structures in the field, however, it is not practical to obtain such free vibration or impulse response.

This paper further develops the nonlinear damping-based damage detection method to extend its application to concrete structures using ambient vibration response, instead of free vibration. In many cases it is relatively easy to obtain ambient vibration responses. Many techniques were developed in the recent years, in order to extract the dynamic characteristics of the structures from ambient vibration responses. They are based on the so-called operational modal analysis, which works with output data under the hypothesis of unknown input excitation [23].

\subsection{RD signature}

Application of the RD signature enables damping analysis of the ambient vibration. The RD method was developed by Cole [3] and used to identify free vibration from a measured random excitation.

The technique is based on averaging of sub-segments extracted from the random signal and chosen with appropriate criteria (referred to as triggering conditions). The averaging procedure results in an $R D$ signature, which minimizes the random component in the original signal by leaving only the response to the triggering conditions. In literature many different triggering conditions can be found [24]. In this study the so-called level crossing triggering condition is employed. Every time the signal crosses the chosen triggering level, a sub-segment is formed. The resulting RD signature is a free decay with initial value equal to the chosen triggering level. The concept can be expressed with the simple formula:

$$
z(\tau)=\frac{1}{N} \sum_{k=1}^{N} y\left(t_{k}+\tau\right)
$$



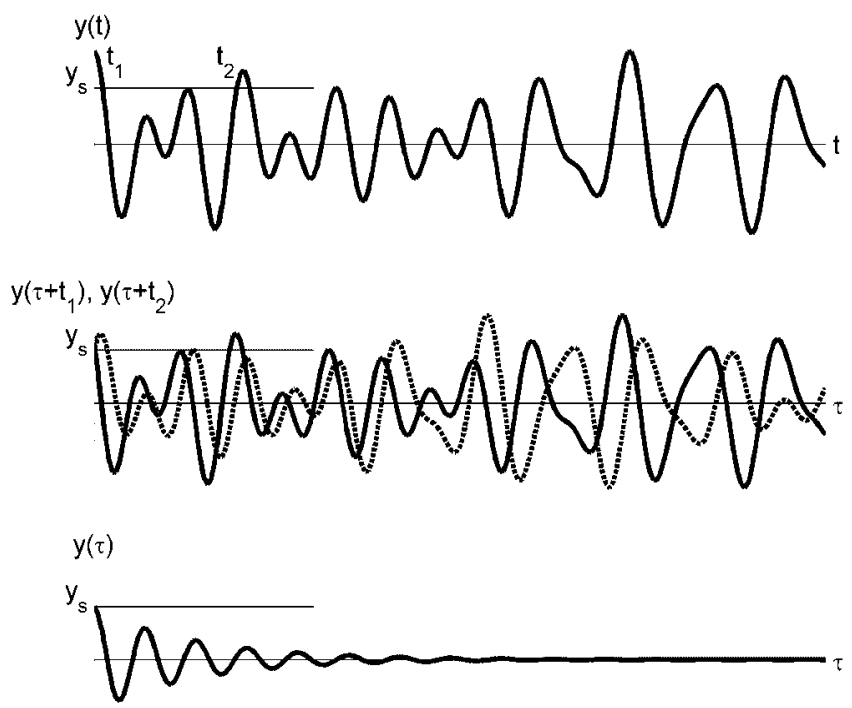

Figure 7. Conceptual extraction of the RD signature from a random response.

where $z(\tau)$ is the RD signature, $\tau$ is the time reference of the sub-segments, $N$ is the number of averages, $y$ is the recoded data, and $t_{k}$ is the time at which the triggering level is crossed. The concept of the algorithm is illustrated in Figure 7.

The triggering level is usually chosen with reference to the standard deviation $\sigma$ of the distribution of the random response. Many studies suggested selecting $\sqrt{2} \sigma$ as the triggering level, since it corresponds to a minimum in the RD signature variance [25].

\subsection{Proposed signal processing approach}

The RD algorithm was tested with a generated response of a SDOF system with known dynamic characteristics, excited with a random force. The response was numerically computed and then processed by the RD algorithm to obtain a free vibration decay, from which the natural frequency and damping were extracted. A good agreement with the theoretical values was observed, but the results showed to be strongly dependent on the length of the sub-segment signals. With sub-segments longer than $20-30 \%$ of the total time window, the random component does not average exactly to zero. Many tests showed that better results were reached with time segments no longer than $5-10 \%$ of the total time window. The best results were obtained by taking the average of the values obtained from more than one random responses. The reason is that if the generated signal is not purely random, the average of its sub-segments cannot go to zero. By taking average of different sets of random responses, the bias contained in a single signal is removed. In addition, the choice of $\sqrt{2} \sigma$ as the triggering level showed to result in the best results.

Figure 8 shows the values of viscous damping calculated with reference to the number of averages. An average of five different sets of white noise (WN) response was also performed. It is observed that the error between the identified and the expected damping ratios decreases as (1) the number of averaging increases, and (2) the data increase from a signal set to five sets. 


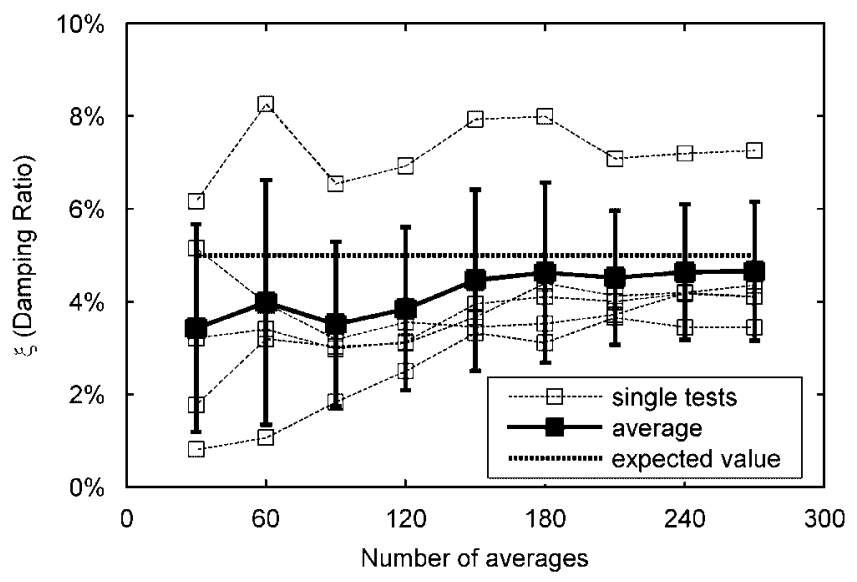

Figure 8 . Test of the RD algorithm: values of $\xi$ identified vs the expected (5\%).

\section{SEISMIC SHAKING TABLE TESTS}

In order to investigate the effectiveness of the proposed nonlinear damping-based damage detection technique, shaking table tests were performed on a large-scale three-bent bridge model at University of Nevada, Reno. In order to accommodate the large size of the bridge, three shaking tables were used. As shown in Figure 9, each of the three bents had two columns and was supported individually by a shaking table. The bridge deck consisted of three posttensioned beams. All the columns had the same design cross sections, but were of different heights, leading to different stiffness. To resemble the inertia of other parts of the superstructure not built into the model, or the inertia from the abutments on both ends, additional masses were added. The shaking tables were driven by input acceleration in the transverse direction. Gaps were provided at the connections between the deck and the bents, so that the deck was divided into two simply supported spans in the vertical direction. However, in the transverse direction the existence of the post-tension tendons and the pre-stressed strain made the three individual beams behave as one beam with a large cross section. Eleven FBA-11 type accelerometers were used to obtain the vibration response of the bridge model in the transverse direction, with their locations indicated in Figure 9.

The model was shaken to different levels of damage by a sequence of earthquake ground motions with increasing intensities. The strong motions were classified into different levels, such as low, moderate, high, severe, and extreme levels. In between these events, low amplitude WN, simulating ambient vibration, drove the shaking tables to perturb the specimen in the corresponding damage level. Table I lists the sequence of the tests and the peak ground acceleration of some representative inputs. The three shaking tables were driven by the identical seismic ground motion signal to produce coherent input. Different levels of damage were observed on the bridge after each strong ground motion. The damage description shown in Table I represents the damage visually observed and confirmed by the strain measurement using strain gauges embedded in steel rebars before concrete casting. During the test, after each strong motion, cracks were marked and photos were taken to document the damage.

Owing to different stiffness of the bents, dynamic behavior was highly dominated by the torsion demanding high transverse movement for the first and the third bent. This explains the 


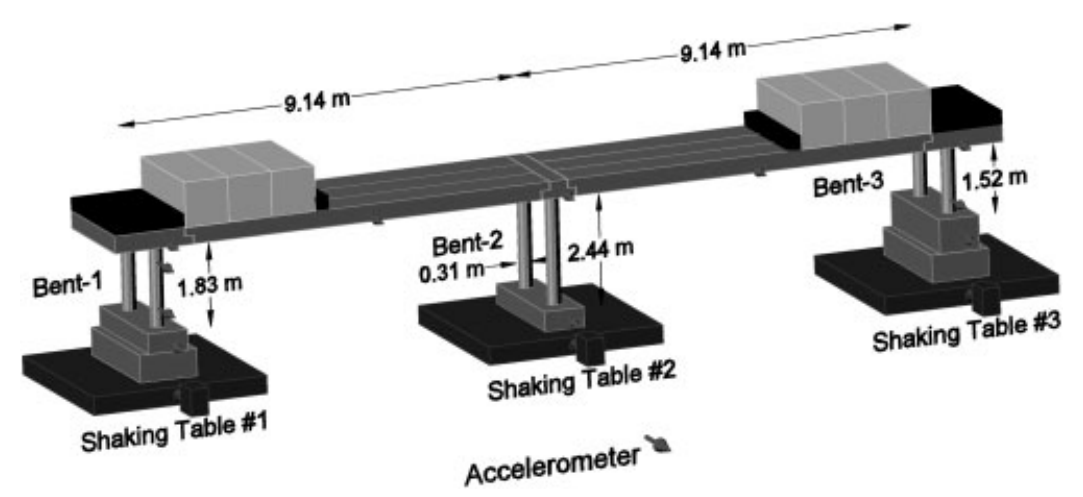

Figure 9. Schematic view of the bridge model and the sensor layout.

Table I. Seismic shaking table test procedure and observed damage.

\begin{tabular}{|c|c|c|c|}
\hline Test & Ground motion description & PGA $(g)$ & Damage description \\
\hline $\mathrm{WN}-1$ & White noise & & \\
\hline $\mathrm{T}-13$ & Low earthquake & 0.17 & Bent-1 yields \\
\hline $\mathrm{T}-14$ & Moderate earthquake & 0.32 & Bent-3 yields \\
\hline $\mathrm{WN}-2$ & White noise & & \\
\hline $\mathrm{T}-15$ & High earthquake & 0.63 & Bent-2 yields \\
\hline WN-3 & White noise & & \\
\hline $\mathrm{T}-19$ & Extreme earthquake & 1.70 & Bent-3 steel buckles \\
\hline WN-4 & White noise & & \\
\hline
\end{tabular}

reason of the severe damage on these two and comparatively lighter damage on the second, middle, bent. The onset of Bent-1 yielding is due to the fact that the first mode of this bridge specimen (at its undamaged stage) has the largest displacement demand on Bent-1. After the yielding of Bent-1, Bent-3 attracts most the seismic force and yields, and then so happens to Bent-2 after the yielding of Bent-1 and Bent-3. The final collapse (in the test, the specimen was protected to avoid actual collapse) is associated with the steel buckling at Bent-3, which has the smallest ductility capacity among the three bents.

\section{DAMAGE IDENTIFICATION}

The proposed nonlinear damping-based damage detection method was verified by the bridge seismic shaking table tests. The damage identification procedure was applied to the responses to the four sets of low-level WN inputs (simulating ambient vibration). The study only used the ambient responses measured at the top of the bents. Those measured at the bottom were strongly contaminated by noise. In addition, the length of sub-segments was changed with reference to the selected $\mathrm{WN}$ in order to pick the same number of oscillation peaks in every case.

Figure 10 shows the procedure applied to the third white noise (WN-3) response of Bent- 1 . It consists in the following four steps: (a) choice of the triggering level with reference to (b) data distribution; (c) application of the RD signature and calculation of the envelope through both positive and negative peak picking; and (d) data fitting with the two described damping models. 

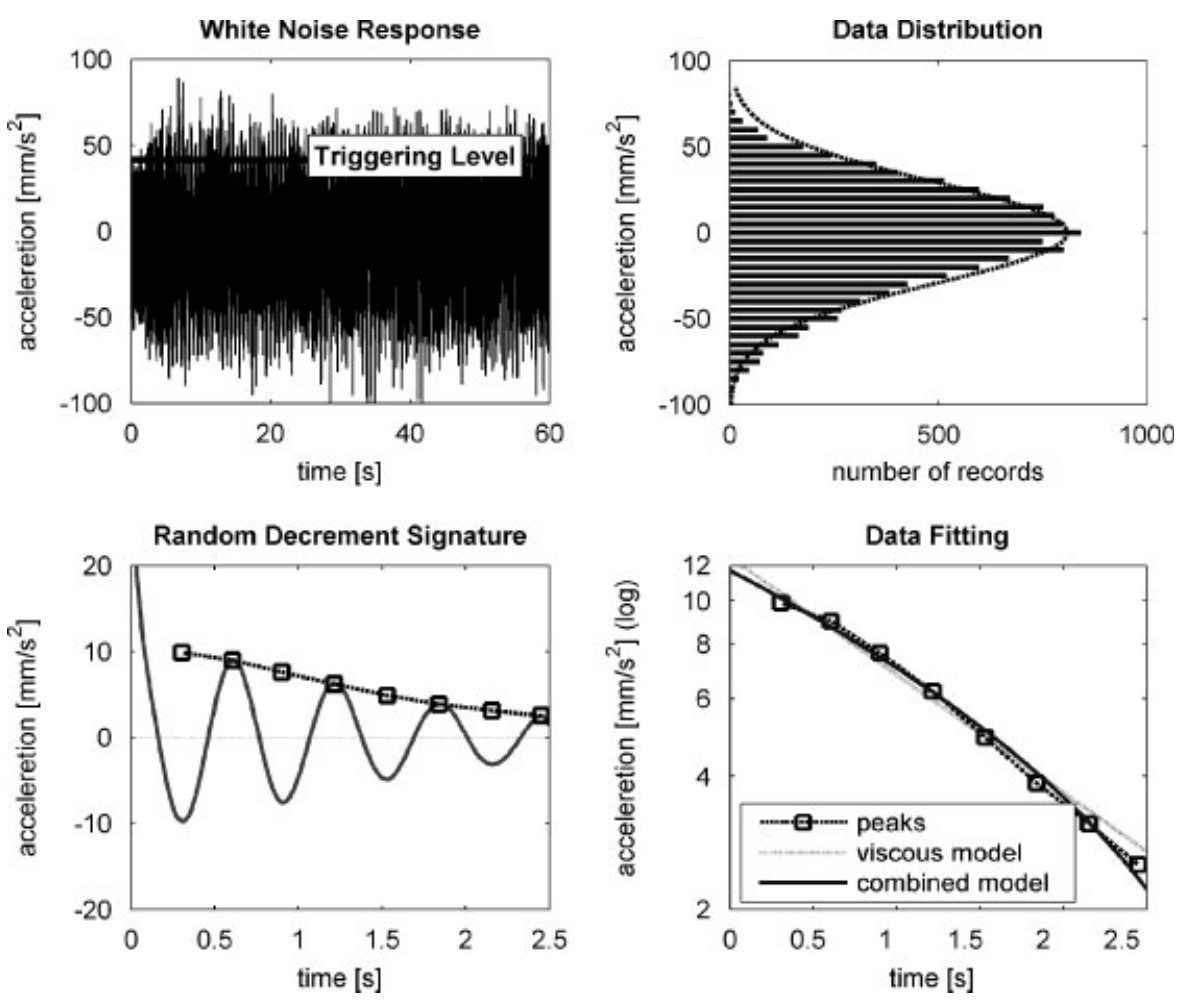

Figure 10. Scheme of the applied procedure.

In the following figures the results based on the Bent-1 measurements are reported for the four WN inputs considered. In Figure 11 the RD signatures obtained from the applied algorithm are shown; the calculated envelopes of the oscillation are also displayed. Figure 12 shows the same curves fitted with the two different models, i.e. the purely viscous model and the viscous-friction combined model. The obtained damping parameters are summarized in Table II, together with the values of the natural frequency obtained from the same RD signatures.

Table II presents the damping identification results based on the two models - the purely viscous model and the viscous-friction combined model. By comparing the root mean square error (RMSE) between the identified and theoretical damping ratios as listed in the table, it is observed that the purely viscous model describes the WN-1 results better than the combined model. In contrast, for WN-3 and WN-4 in which the bridge is damaged, the combined model fits better the envelopes than the purely viscous damping model, as demonstrated from their RMSE values. Overall the viscous-friction combined model fits better with the results identified from the measurement, particularly when the bridge suffers more severe damage.

Figure 13 plots the viscous and friction damping ratios in the combined model. Recall that the four sets of WN (ambient vibration) responses were measured at the bridge with different levels of seismic damage as shown in Table I, with no damage in $\mathrm{WN}-1$ to severe damage in WN-4. Therefore, the $X$-axis also represents the severity of the seismic damage. As the seismic damage becomes more severe, the viscous damping ratio $\xi$ decreases, while the frictional 

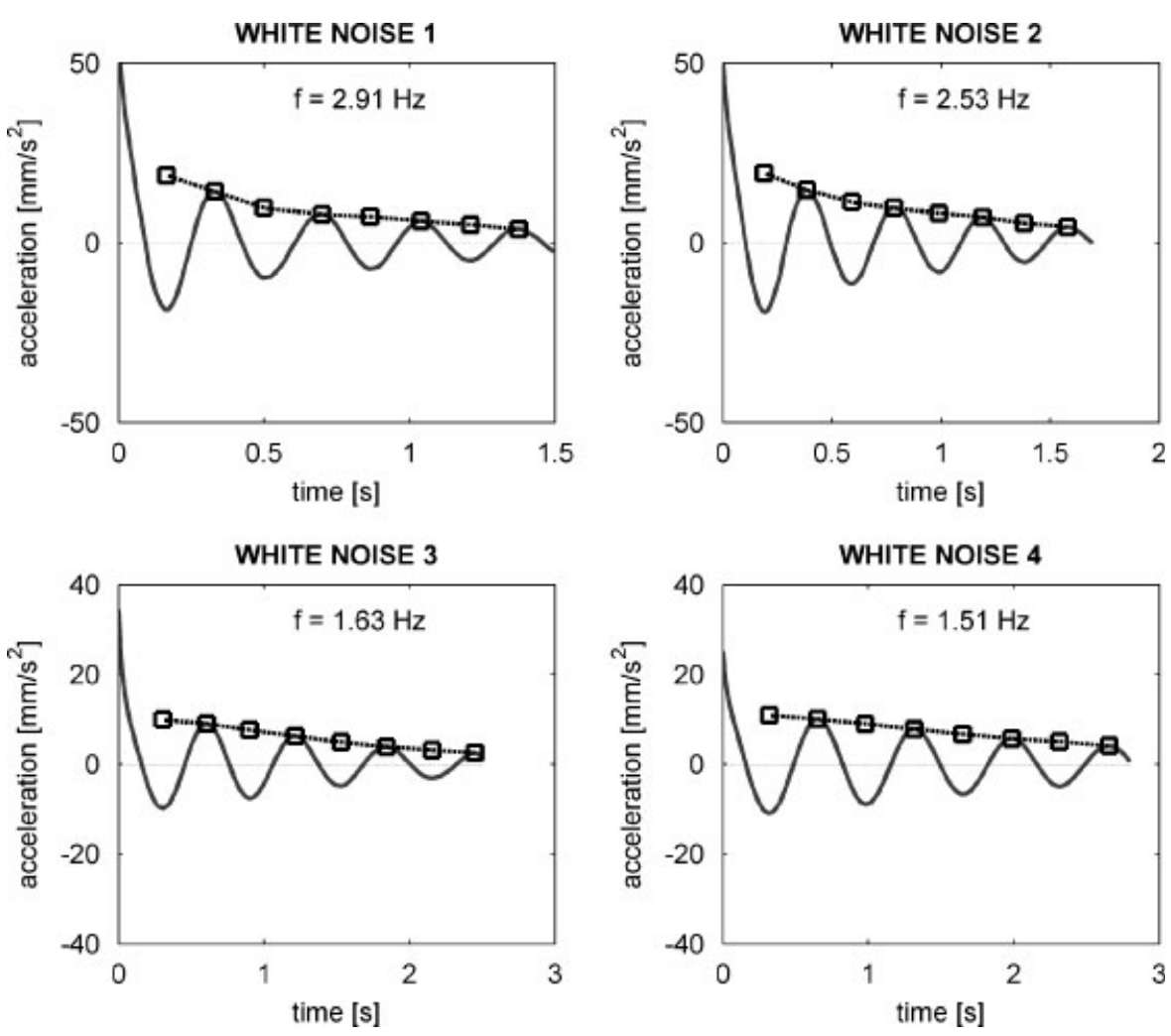

Figure 11. RD signature and envelopes of oscillation.

damping ratio $\gamma$ increases. As mentioned above, the values of $\gamma$ and $\xi$ provide information about the percentage of the total energy dissipated by each damping phenomenon. Following this interpretation it can be concluded that there is a shift of dissipated energy from a viscous mechanism (material damping) to a friction mechanism (crack damping) with the evolution of damage. The presence of cracks in the structure is the direct cause of the development of a friction mechanism, which can be therefore assumed as a reliable damage index.

The seismic damage identified based on the nonlinear damping analysis shown in Figure 13 was consistent with the observed damage described in Table I that was based on the strain measurement at the column rebar as well as visual inspection. Therefore, the effectiveness of the proposed nonlinear damping-based damage identification method was experimentally verified.

The nonlinear damping parameter $\gamma$ can serve as a damage index. When its value equals zero, it means no damage. A significant advantage of this damage index is its baseline-free feature. The damage identification can be performed using only one set of ambient vibration measurement without comparison with a non-damaged state. For example, if only WN-4 is measured at the bridge in the seismic shaking table tests, the bridge structure can be identified as damaged, because the value of $\gamma$ is larger than zero. Similarly, from the results of WN-1, the structure can be considered undamaged, because there is no energy dissipated by the friction mechanism. The anomaly itself identifies the damage. 

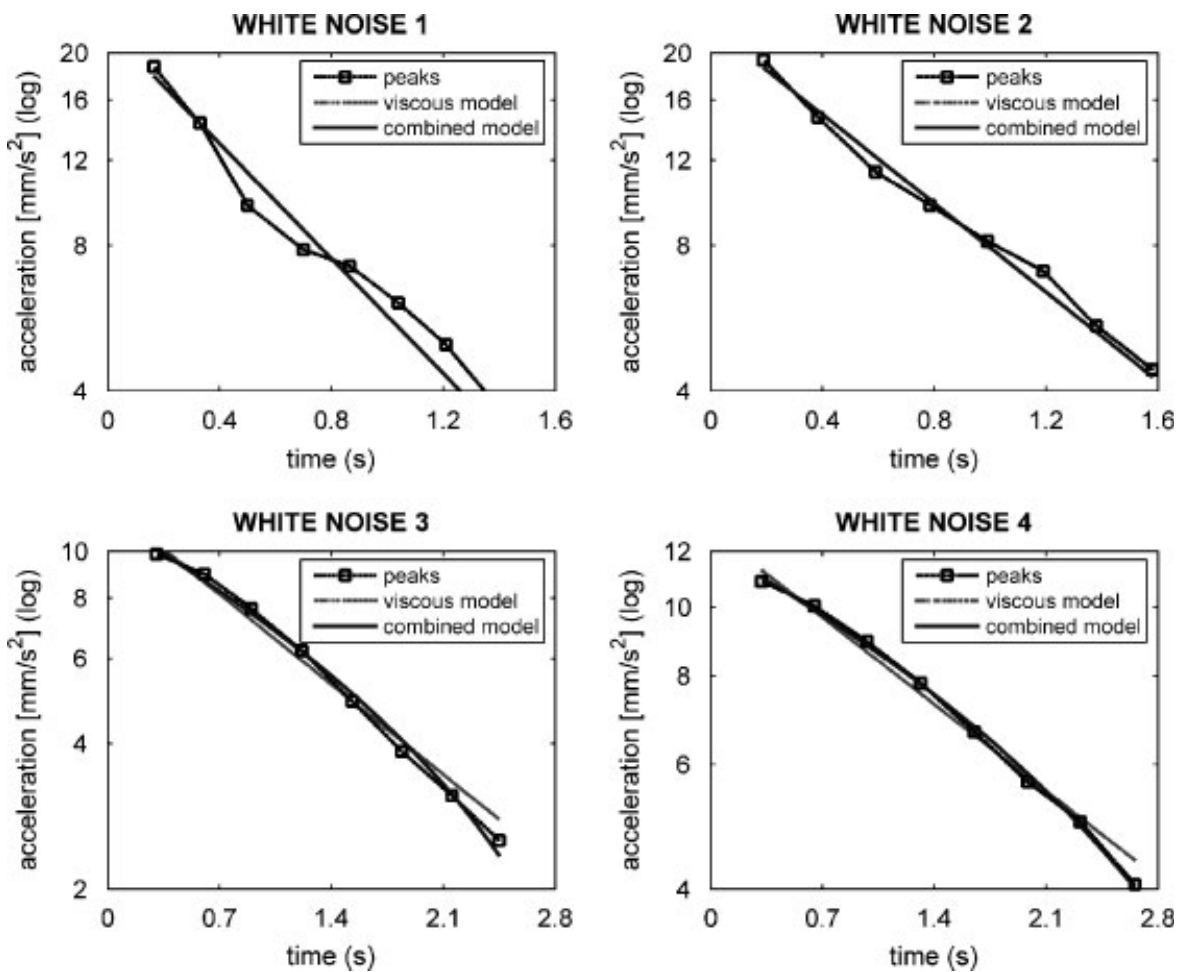

Figure 12. Fitting of the envelopes with the two described models.

Table II. Damping parameters calculated with the two described models and corresponding RMSE.

\begin{tabular}{lcccc}
\hline & White noise 1 & White noise 2 & White noise 3 & White noise 4 \\
\hline Frequency (Hz) & 2.91 & 2.53 & 1.63 & 1.51 \\
$\xi$ (viscous) & 0.074 & 0.066 & 0.056 & 0.041 \\
RMSE (viscous) & $9.32 \mathrm{e}-4$ & $5.61 \mathrm{e}-4$ & $3.59 \mathrm{e}-4$ & $2.82 \mathrm{e}-4$ \\
$\xi$ (combined) & 0.074 & 0.066 & 0.028 & 0.013 \\
$\gamma$ (combined) & 0.000 & 0.000 & 0.014 & 0.017 \\
RMSE (combined) & $1.10 \mathrm{e}-3$ & $6.59 \mathrm{e}-4$ & $2.53 \mathrm{e}-4$ & $1.46 \mathrm{e}-4$ \\
\hline
\end{tabular}

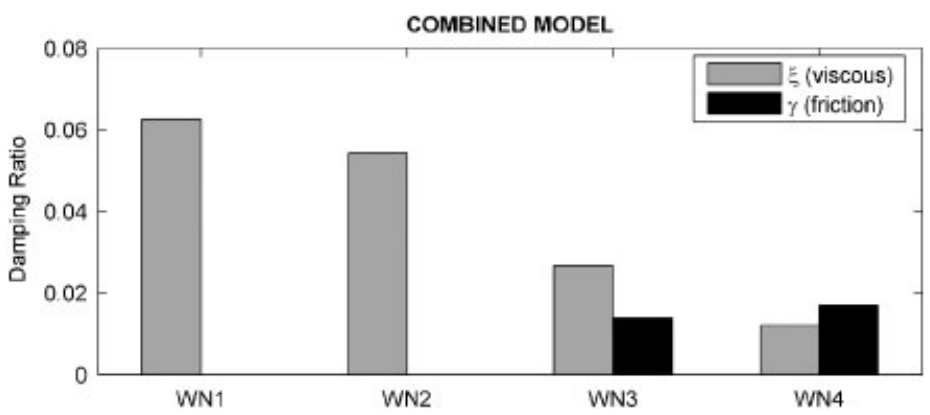

Figure 13. Comparison of the damping parameters. 


\section{COMPARISON WITH STIFFNESS REDUCTION}

The identified change in the friction characteristics associated with the seismic damage was further compared with the change in structural stiffness. For this purpose, hysteretic loops were obtained from the RD signatures. The RD acceleration signals were double integrated to obtain the RD displacements. The plots, as shown in Figure 14, use the measured acceleration under the assumption that the mass of the bridge is constant during the damaging events. It is observed that the structural stiffness decreases as the seismic damage becomes more severe. The $\mathrm{RD}$ algorithm proved to be a simple way to analyze the stiffness degradation associated with seismic damage.

Furthermore, the damage identification results obtained in this study were compared with those using structural stiffness as a damage index. Different damage detection methods based on structural stiffness were proposed by the same group of researchers and verified by the same bridge seismic shaking table test results, as reported in [17,18]. In [17], an extended Kalman filter was applied to analyze the seismic response data and to instantaneously calculate the reduction of stiffness in each bent caused by the seismic damage. In [18], stiffness reduction was identified by processing the pre- and post-event WN responses based on a linear timeinvariant model.
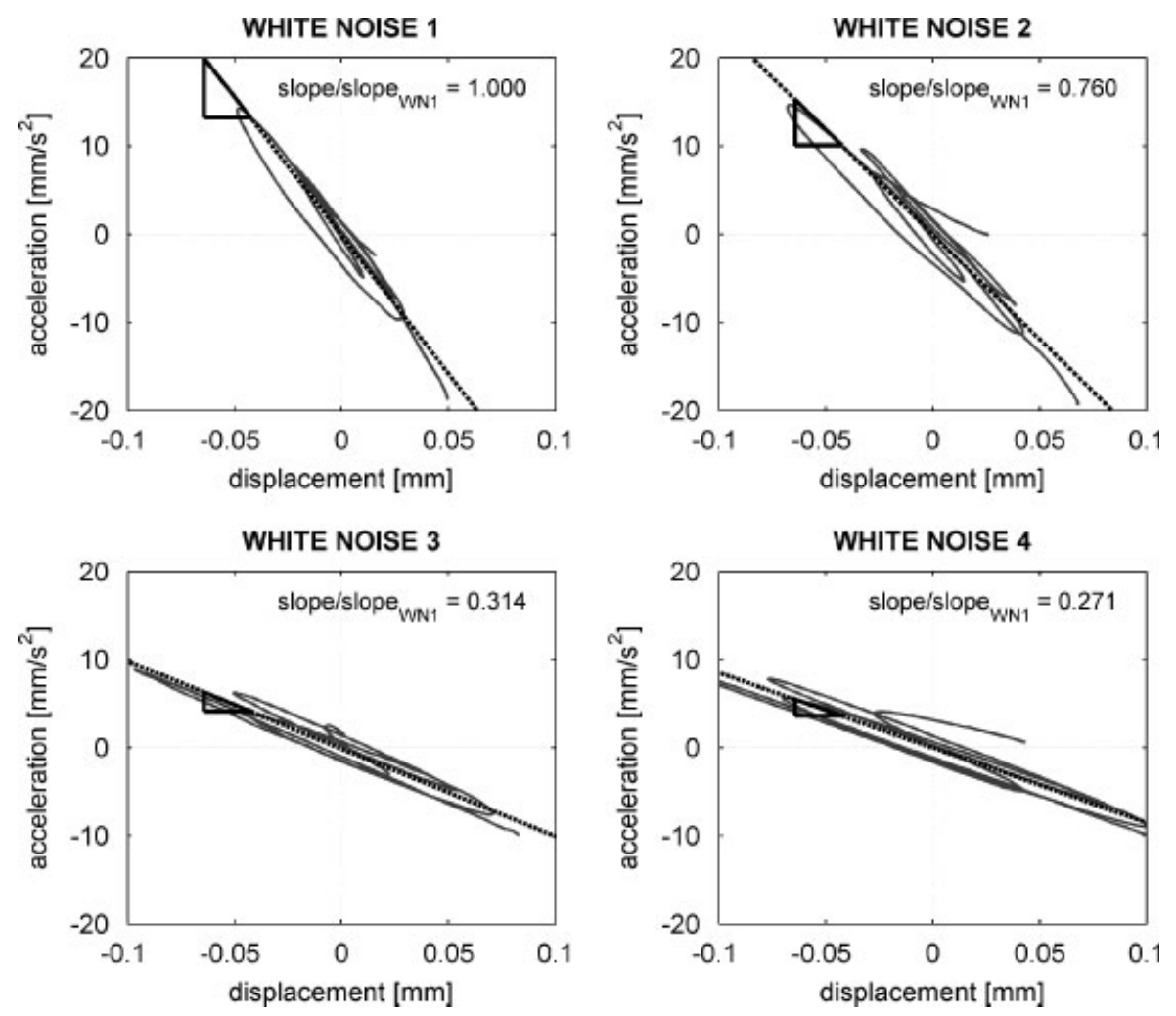

Figure 14. Hysteretic loops from the random decrement signatures. 


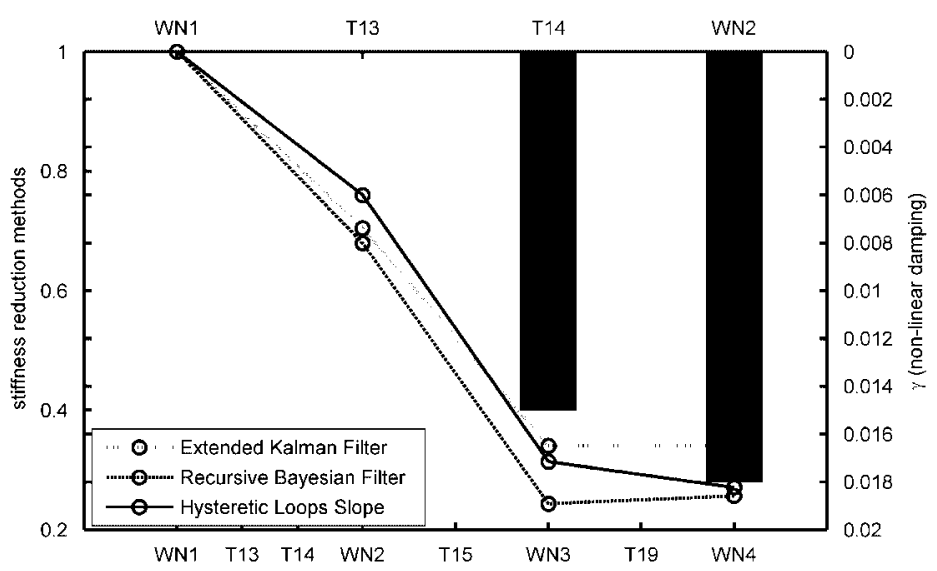

Figure 15. Comparison of damage identification results based on damping and stiffness.

Figure 15 summarizes the identified stiffness reduction results obtained in the previous studies, compared with the ones calculated through the hysteretic loops in Figure 14 and through the nonlinear damping method in Figure 13. The stiffness is measured by the $Y$-axis on the left side of the figure, normalized with the stiffness values computed in $\mathrm{WN}-1$. As the results, the stiffness values start from 1.0 , implying $100 \%$ of the total stiffness when the structure is undamaged. The stiffness values identified by the three different methods all decrease as the seismic damage becomes more severe. The nonlinear damping ratio is presented by the $Y$-axis on the right side of the figure. The damping increases as the seismic damage becomes more severe. The figure shows a reasonably strong correlation between the nonlinear damping ratio $\gamma$ and the stiffness reduction associated with different levels of seismic damage on the bridge, particularly for the heavily damaged cases (WN-3 and WN-4). In summary, the damage identification results from the four different damage identification methods show a good agreement.

\section{CONCLUSIONS}

In this paper a damage detection method was developed for reinforced concrete structures based on the analysis of a damage index expressed as the nonlinear damping ratio. The method was applied for the first time for detecting seismic damage in a concrete structure, a realistic bridge model subjected to realistic seismic damage. The RD signature technique is employed to process measured structural responses to ambient vibration, in order to facilitate the identification of the nonlinear damping ratio. Different levels of seismic damage on the bridge model were successfully identified by the nonlinear damping-based method. The following conclusions can be made.

(a) The nonlinear damping parameter $\gamma$ proved to be a reliable damage index, as it is not only highly sensitive to seismic damage of different levels, but also consistent with the observed damage.

(b) A major advantage of the proposed nonlinear damping-based damage detection method is its baseline-free feature. The nonlinear damping parameter is able to detect the presence of damage in the structure without any reference to the undamaged baseline. 
(c) A strong correlation is observed between the increase in the nonlinear damping and decrease in the structural stiffness associated the increase in seismic damage severity.

\section{ACKNOWLEDGEMENTS}

This work is sponsored by the California Department of Transportation (Caltrans) under contract 59A0311 and Federal Highway Administration under contract DTFH61-07-C- 00031. The advice and assistance from $\mathrm{Mr}$ Lihong Sheng at the Caltrans are highly appreciated. The authors would also like to express sincere gratitude to Professors M. Saiid Saiidi and D. Sanders at University of Nevada, Reno, for providing an opportunity to collaborate on the shaking table experiments.

\section{REFERENCES}

1. Cawley P, Adams RD. The location of defects in structures from measurements of natural frequencies. Journal of Strain Analysis 1979; 14(2):49-57.

2. Hunt DL, Weiss SP, West WM, Dunlap TA, Freemeyer SR. Development and implementation of a shuttle modal inspection system. Journal of Sound and Vibration 1990; 24(9):34-42.

3. Cole A. On-line failure detection and damping measurement of aerospace structures by random decrement signature. NASA CR 2205, 1979.

4. Farrar CR, Jauregui DA. Comparative study of damage identification algorithms applied to a bridge: I. Experiment. Smart Material and Structures 1998; 7:704-719.

5. Farrar CR, Jauregui DA. Comparative study of damage identification algorithms applied to a bridge: II. Numerical Study. Smart Materials and Structures 1998; 7:720-731.

6. Doebling SW, Farrar CR, Prime MB, Shevitz DW. Damage identification and health monitoring of structural and mechanical systems from changes in their vibration characteristics: a literature review. Report LA-13070-MS, Los Alamos National Laboratory, 1996.

7. Salawu OS. Detection of structural damage through changes in frequency: a review. Engineering Structures 1997; 19(9):718-723.

8. Alvandi A, Cremona C. Detection of vibration-based damage identification techniques. Journal of Sound and Vibration 2005; 292:179-202.

9. Ghanem R, Shinozuka M. Structural system identification I: theory. Journal of Engineering Mechanics 1995; 121(2):255-264.

10. Shinozuka M, Ghanem R. Structural system identification II: experimental verification. Journal of Engineering Mechanics 1995; 121(2):265-273.

11. Neild SA, Williams MS, McFadden PD. Nonlinear vibration characteristics of damaged concrete beams. Journal of Structural Engineering 2003; 129(2):260-268.

12. Stubbs N, Kim JT. Damage localization in structures without baseline modal parameters. AIAA Journal 1996; 34(8): 1644-1649.

13. Franchetti $\mathrm{P}$, Modena $\mathrm{C}$, Feng MQ. Nonlinear damping identification in precast prestressed reinforced concrete beams. Computer-Aided Civil and Infrastructure Engineering, submitted for publication.

14. Modena C, Sonda D, Zonta D. Damage localisation in reinforced concrete structures by using damping measurements. Key Engineering Materials 1999; 167-168:132-141.

15. Franchetti P. Vibration-based damage detection of precast elements. Ph.D. Dissertation, DIMS, Università degli Studi, Trento, Italy, 2004.

16. Zonta D. Structural damage detection and localization by using vibrational measurements. Ph.D. Dissertation, DISTART, Università degli Studi, Bologna, Italy, 2000.

17. Soyoz S, Feng MQ. Seismic damage detection based on structural stiffness and experimental verification. Structural Control and Health Monitoring 2008; 15(7):958-973.

18. Chen Y, Feng MQ, Soyoz S. Large-scale shaking table test verification of bridge condition assessment methods. ASCE Journal of Structural Engineering 2008; 134(7):1235-1245.

19. Mahrenholtz O, Bachmann H. Appendix C-damping. Comité Euro- International du Beton-CEB, Lausanne, Switzerland, vol. 209, 1991; 169-180. 
20. Bachmann H, Dieterle R. Experiments and models for the damping behavior of vibrating reinforced concrete beams in the uncracked and cracked conditions. Bericht Nr. 119, Institute of Structural Engineering ETH, Zurich, 1981.

21. Konig G, Fehling E. Excitation damping of reinforced concrete under cycle. XIII IABSE Colloquium, Helsinki, 1989; 359-664.

22. Tomlinson GR, Hibbert JH. Identification of the dynamic characteristics of a structure with coulomb friction. Journal of Sound and Vibration 1979; 64(2):233-242.

23. Brincker R, Zhang L, Andersen P. Modal identification of output-only systems using frequency domain decomposition. Smart Material and Structures 2001; 10:441-445.

24. Asmussen JC. Modal analysis based on the random decrement technique. Ph.D. Dissertation, Department of Building Technology and Structural Engineering, University of Aalborg, Denmark, 1997.

25. Asmussen JC, Brincker R, Ibrahim SR. Statistical theory of the vector random decrement technique. Journal of Sound and Vibration 1999; 226(2):329-344. 